\title{
Proceedings of the 2017 GRAPPA Collaborative Research Network Meeting
}

\author{
Deepak R. Jadon, Vinod Chandran, Carmel Stober, Alexis Ogdie, April W. Armstrong, \\ Kristina Callis Duffin, Dafna D. Gladman, Philip S. Helliwell, Denis O'Sullivan, \\ Maarten de Wit, Oliver FitzGerald, and Christopher T. Ritchlin
}

\begin{abstract}
The Group for Research and Assessment of Psoriasis and Psoriatic Arthritis (GRAPPA) Collaborative Research Network (CRN) is an endeavor that aims to address gaps in the knowledge of the etiopathogenesis and management of psoriatic disease by best using the large community of experienced investigators who are already collecting rich clinical phenotype data and biologic samples using validated techniques. Exemplar rheumatology and dermatology projects will inform strategies to implement the CRN, while input and funding from government organizations, charities, and industry will shape the CRN. The key immediate priorities to establish the CRN are discussed herein and include (1) strategies for building infrastructure to collect and store biosamples and associated clinical data, (2) best practices for sample collection and storage, (3) approaches to engage the GRAPPA community of investigators and industry to collaborate most effectively on shared priorities, and (4) agreement on a funding strategy. The following 4 CRN candidate flagship research areas were identified: (1) predictors of treatment response in psoriatic arthritis (PsA) and cutaneous psoriasis (PsC) to permit personalized and stratified medicine approaches; (2) predictors of structural damage and disease severity, linking with the existing PsA BioDAM project; (3) predictors of PsC progressing to PsA to enable earlier intervention and possibly halt progression to PsA; and (4) comorbidity prevalence and effect on clinical outcomes in psoriatic disease. The collaboration and momentum provided by a GRAPPA-CRN will offer more than the sum of its individual contributing centers. ACRN will permit high-quality research that can more effectively address questions pertinent to patients, clinicians, scientists, industry, and governments. (J Rheumatol Suppl. 2018 June;94:54-61; doi:10.3899/ jrheum.180141)
\end{abstract}

Key Indexing Terms: PSORIATIC ARTHRITIS RESEARCH

IMAGING

PSORIASIS

the Netherlands. The CRN meeting was organized by a committee co-chaired by Professors Oliver FitzGerald and Christopher T. Ritchlin. The meeting was attended by 30 rheumatologists, 4 dermatologists, 11 leads from the pharma-

\section{BIOMARKERS OUTCOME MEASURES}

The Group for Research and Assessment of Psoriasis and Psoriatic Arthritis (GRAPPA) Collaborative Research Network (CRN) held its inaugural meeting over 2 days following the GRAPPA 2017 annual meeting in Amsterdam,

\begin{abstract}
From the Rheumatology Research Unit, Cambridge University Hospitals UK National Health Service (NHS) Foundation Trust, Cambridge; University of Leeds, Leeds; Bradford Hospitals NHS Foundation Trust, Bradford, UK; Department of Medicine, Division of Rheumatology, University of Toronto, Toronto Western Hospital, Krembil Research Institute, Toronto, Ontario, Canada; Perelman School of Medicine, University of Pennsylvania, Philadelphia, Pennsylvania; University of Southern California, Los Angeles, California; University of Utah, Salt Lake City, Utah; Division of Allergy, Immunology, and Rheumatology, University of Rochester Medical Center, Rochester, New York, USA; Our Lady's Hospice and Care Services; Newman Clinical Research Professor, Department of Rheumatology, St. Vincent's University Hospital and Conway Institute for Biomolecular Research, University College Dublin, Dublin, Ireland; Department of Medical Humanities, VU University Medical Centre, Amsterdam, the Netherlands.
\end{abstract}

As part of the supplement series GRAPPA 2017, this report was reviewed internally and approved by the Guest Editors for integrity, accuracy, and consistency with scientific and ethical standards.

D.R. Jadon, MBBCh, MRCP, PhD, Director of Rheumatology Research Unit, Cambridge University Hospitals NHS Foundation Trust;

V. Chandran, MBBS, MD, DM, PhD, Department of Medicine, Division of Rheumatology, University of Toronto, Toronto Western Hospital, Krembil
Research Institute; C. Stober, PhD, MBChB, MRCP, Consultant Rheumatologist, Cambridge University Hospitals NHS Foundation Trust; A. Ogdie, MD, Assistant Professor of Medicine and Epidemiology, Perelman School of Medicine, University of Pennsylvania;

A.W. Armstrong, MD, MPH, University of Southern California; K. Callis Duffin, MD, University of Utah; D.D. Gladman, MD, FRCPC, Professor of Medicine, University of Toronto, and Senior Scientist, Krembil Research Institute, Toronto Western Hospital; P.S. Helliwell, DM, PhD, FRCP, University of Leeds, and Bradford Hospitals NHS Foundation Trust; D. O'Sullivan, BE, Patient Research Partner, Our Lady's Hospice and Care Services; M. de Wit, PhD, Patient Research Partner, Department of Medical Humanities, VU University Medical Centre; O. FitzGerald, MD, FRCPI, FRCP $(U K)$, Newman Clinical Research Professor, Department of Rheumatology, St. Vincent's University Hospital and Conway Institute for Biomolecular Research, University College Dublin; C.T. Ritchlin, MD, MPH, Professor of Medicine, Division of Allergy, Immunology, and Rheumatology, University of Rochester Medical Center.

Professor FitzGerald and Professor Ritchlin are joint senior authors.

Address correspondence to Dr. D.R. Jadon, Director of Rheumatology Research Unit, Cambridge University Hospitals NHSFT,

Addenbrooke's Hospital, Hills Road, Cambridge, CB2 OQQ, UK.

E-mail:deepak.jadon@addenbrookes.nhs.uk 
ceutical industry, 6 patient research partners (PRP), and 2 nonmedical scientists.

Several motivating factors converged to catalyze the establishment of a GRAPPA CRN. Foremost are major gaps in our knowledge of the etiopathogenesis of psoriatic disease, coupled with the emergence of a large community of experienced investigators already collecting rich clinical phenotype data and biologic samples using validated standardized operating procedures (SOP). Another factor is the successes of previous and existing research efforts in this and related areas, e.g., the psoriatic arthritis (PsA) BioDAM project, rheumatoid arthritis (RA) BioDAM project, and the International Psoriasis and Arthritis Research Team (IPART). There also appears to be a willingness and building momentum from national organizations such as the Innovative Medicines Initiative (IMI), US National Institutes for Health (NIH), and the Accelerating Medicines Partnership (AMP) ${ }^{1}$ to support such collaborations or serve as models for data collection and analysis. GRAPPA has a growing membership of over 400 rheumatologists and dermatologists. GRAPPA's community of young investigators and trainees is a precious resource, as evidenced by the Trainee Symposium at the GRAPPA 2017 annual meeting, in which 40 high-quality abstracts were submitted, translating to 6 oral and 23 poster presentations. Further, GRAPPA received 23 research grant applications in 2017, and 5 grants were awarded.

Over the last year, the GRAPPA Research and Biomarkers Committee has generated momentum for the CRN and spearheaded this meeting with the following key objectives: (1) to decide on an overall strategy for building infrastructure to collect and store biosamples and associated clinical data; (2) to determine best practices for sample collection and storage; (3) to identify approaches to engage the GRAPPA community of investigators to participate with the CRN; (4) to understand how to optimize collaborative opportunities between the CRN and industry partners; and (5) to agree on a feasible strategy to fund the CRN.

Some key questions and challenges in setting up the CRN were also identified by the committee for further examination at this meeting. The first was whether samples should be stored centrally at sampling centers, or a mixed-model approach should be used, with some samples stored at local sites and others in a central repository. The CRN must identify and address barriers to biosamples and data crossing international borders. A strategy is required to optimally engage investigators to contribute samples and research proposals, and industry partners on shared research priorities and financial support. The overarching challenge, but ultimately the reward, is how best to make the CRN's sum greater than its individual parts.

\section{Gaps and Emerging Opportunities in PsA}

The NIH National Institute of Arthritis and Musculoskeletal and Skin Diseases (NIAMS) reported their 2017 "Roundtable on Gaps and Emerging Opportunities in Psoriatic Arthritis"2. Several priority areas were identified. A better understanding is needed of the pathogenesis of PsA, including genetic, epigenetic, and environmental factors, as well as the differences and similarities between PsA and related conditions. Advancement of translational research through better-resourced research and using PsA as a model for understanding preclinical autoimmunity was stated as a priority. Clinical research and new therapies research is needed to permit personalized treatment approaches for PsA, to identify biomarkers to facilitate diagnosis and treatment, and to better address comorbidities. NIAMS also called for better engagement of patients in clinical research and trials, while also attracting and retaining clinical researchers.

\section{AMP}

The AMP, particularly its "Autoimmune Diseases of Rheumatoid Arthritis and Lupus Network"3 , was cited as an example of how multiple sites across the United States can be set up and co-funded through the NIH and industry. As well as co-funding, industry partners are actively participating and guiding the network's scientific direction. Peripheral blood mononuclear cells (PBMC) and tissues (synovial, skin, and renal tissue) are being collected and analyzed using sophisticated techniques such as single-cell RNA sequencing, CyTOF (mass cytometry), and laser capture microdissection. Centralized storage and SOP have been an integral component of the network. The network has benefited from a $\$ 41.6$ million fund over 5 years, with $\$ 20.9$ million from the NIH, \$20.7 million from industry, and $\$ 0.3$ million from nonprofit organizations.

\section{IPART}

The IPART initiative was described by Professor Dafna D. Gladman. Funded by the Canadian Institutes of Health Research until 2012, and subsequently by unrestricted grants from industry, its objectives have been to form a database of well-characterized cases (clinically, radiologically, and laboratory) to identify susceptibility factors for PsA among psoriasis-only (PsC) cases and risk factors for disease severity. A shared Internet-based database and rigorous multicenter clinical skills training underpins the effort. The model of a multicenter clinical trial was used to attain ethical approval at the different centers. Each contributing center keeps ownership of its entered data and is able to view its data. Potential collaborative studies are presented at the IPART annual meeting, and with agreement, sites release specific datasets. Only centers contributing data for a specific paper are included in the authorship. Several challenges for IPART were identified, including recruitment to yearly blood sampling, high attrition among younger patients, and the need for a research assistant supervised by the database manager to ensure complete data. Because maintenance costs have amounted to $\$ 0.5 \mathrm{million} /$ year (administrator, database

Personal non-commercial use only. The Journal of Rheumatology Copyright (C) 2018. All rights reserved. 
manager, research assistants, sampling equipment), it is recommended that these are included in project grants.

\section{RA BioDAM}

Professor Walter Maksymowych gave an overview of the RA BioDAM project. This project was set up using the "clinical trial" model (rather than the "registry" model) and uses the biorepository platform with linked clinical data. Its objective was to determine a serum-soluble biomarker that would predict prognosis, making imaging and other laboratory tests unnecessary. This 2-year prospective observational cohort recruited 576 RA cases between 2011 and 2017 from 10 countries (Canada, Denmark, France, Germany, Ireland, Israel, Italy, the Netherlands, Norway, and the United States), with a mean followup period of 21 months. Patients were assessed and sampled for both serum and urine every 3 months. All centers signed a contract similar to contracts used in randomized control trials (RCT) to deliver outcomes pursuant to the protocol. The initiative was funded by an unrestricted grant from AbbVie. The key challenges for the RA BioDam project included the acquisition of fasting samples; case attrition of $25 \%$ over 2 years; imaging not always anonymized; imaging not submitted in a timely manner or in the correct format; linking radiographs to the electronic case report forms (eCRF); insufficient serum or urine volumes; and escalating costs, especially for laboratory consumables, salaries, and couriers.

The key recommendations for the CRN committee were to run the CRN similar to an RCT; implement an eCRF-based integration of imaging, biosample record, and shipment date; strictly adhere to validated SOP; courier samples in batches only with complete eCRF; notify customs officials in advance; incorporate a proactive platform for highly responsive quality assurance and query platform for radiography and biosamples; and incorporate realistic recruitment timelines to permit the accurate estimation of study costs.

\section{Perspectives from Industry}

Professor Paul-Peter Tak represented GlaxoSmithKline to give an industry perspective on the CRN. Several key advantages to a CRN approach were reinforced, such as a large well-characterized longitudinal cohort providing betterpowered studies, collaboration of expertise using various platforms, and the integrated development of research methods.

It was recommended that the CRN consider and devise strategies to address financial obligations; overlap with other bioresource initiatives, contracts, confidentiality, and intellectual property (IP) rights; definitions of obligations and responsibilities; resourcing, timelines, and longterm sustainability; sample governance; and maintaining the engagement of contributing centers. Samples likely to be of interest to industry would be blood and synovial tissue both before and after an intervention, with associated clinical data on response versus nonresponse. The right culture should be created from the outset, with a clear and agreed vision, open exchange of expectations, and acknowledgment of geographical and functional differences or priorities. Through strong leadership and clearly apportioned responsibilities, the CRN should aim to develop a robust and accountable governance structure that includes public and private partners. An important component of governance would be the life cycle of a sample: sourcing, use, storage, further use (ownership or custodian), transport, and disposal. Choosing government or public partners, in addition to private partners, would provide more stable funding and permit studies with longer timelines. Examples of public partners to consider included IMI, NIH, AMP, and from the United Kingdom the Medical Research Council (MRC), National Institute for Health Research (NIHR), Wellcome Trust, Francis-Crick Institute, Altius Institute, and the European Bioinformatics Institute. To allow for better experimental design, the full dataset being held centrally without local researcher access was advocated. The differences in ethics, consent, and data privacy across international borders was re-emphasized. It was recommended that the CRN register with the "BioBank Directory" to attract potential users and contributors. The early dissemination of emerging study results to patients and healthcare professionals is good clinical practice, but would also motivate existing sites to recruit, new sites to join, and attract new funders.

\section{Roundtable Discussions}

Fueled by the experience of these initial sessions, a roundtable discussion was undertaken to identify further areas for debate. Specific pertinent research questions are needed as the basis for funding applications. There was an appetite to maximize collaboration between existing cohorts and apply for funding to enable new smaller centers to join. Siting hubs in geographic regions, e.g., European, North American, South American, Asian, African, and pan-Pacific, might help with the ethics and regulations associated with transferring biosamples across borders. Centers storing their own samples initially, and only later developing a central biorepository, may also help. However, this must be balanced against the ability to reproduce laboratory techniques based on validated SOP from academic centers, which might advocate a centralized processing approach for certain types of samples from the outset. Given the expense, space, and associated infrastructure required by the centralized storage of biosamples, it is essential to have generous financial planning and the justification of storage duration. Given the logistical challenges experienced by IPART and RA BioDAM, strong consideration should be given to employing a contract research organization to perform logistics for the $\mathrm{CRN}$, as is done for large late-phase multinational commercial trials.

Consideration should be given to developing an electronic consent form, to enable easier tracking and ensure disposal

Personal non-commercial use only. The Journal of Rheumatology Copyright @ 2018. All rights reserved. 
of data/biosamples if required. Patients withdrawing from the CRN should be given the choice to either have existing data/biosamples destroyed or continue to be used. Such a multinational effort must also accommodate the varying literacy levels and languages used by patients in the multiple centers. Given that the legal framework for GRAPPA currently resides in Seattle (USA), further investigation is needed to determine the effect of a multinational CRN on applying for grants from other geographic areas and on indemnity. Involvement of international lawyers is now therefore needed, at CRN inception, albeit aiming to keep the legal framework as simple as possible. During this inception phase, a longterm strategy for continuous active involvement of PRP is essential. Involvement of PRP at steering committee and subcommittee meetings, to write newsletters for patients, to annually present information to other patients, to review grants from inception, and to contribute to lay writing will add great value to the CRN.

\section{Priority Areas in Psoriatic Disease Research}

Drs. Vinod Chandran and April W. Armstrong identified key areas for PsA and PsC research, respectively (Table 1). They stated that identifying a flagship project that is pertinent to both PsA and PsC and that best uses the existing strengths of GRAPPA members is critical. Similarly, reporting data from the CRN in a clinically meaningful way and using innovative analytic approaches will strengthen this endeavor. The need for systematic timepoints for the biosampling of both lone and combined biomarkers was emphasized. The Vectra-DA project to identify biomarkers that predict disease activity and joint damage in RA (multibiomarker disease activity) was cited as a good example of academic and industry collaboration ${ }^{4}$.

Table 1. Possible flagship research studies for the CRN.

1) Clinical, blood, and/or imaging biomarkers of:

(i) arthritis/skin activity (subclinical, clinical, or response to treatment)

(ii) structural damage and disease severity

(iii) global inflammatory burden

(iv) response to treatment

(v) adverse events

(vi) development of PsA phenotypes (e.g., PsA mutilans or psoriatic spondyloarthritis)

2) Modifying outcomes from comorbid diseases (e.g., through smoking cessation, weight-reduction strategies, diet, lifestyle, statins, metformin, and antihypertensives)

3) Health-related quality-of-life markers

4) Predictors of $\mathrm{PsC}$ progressing to PsA

5) Predictors of patients with a family history, but currently no personal history, of PsC or PsA, developing psoriatic disease

6) Development of a diagnostic kit for PsC and PsA

7) Molecular classification of psoriatic disease

CRN: collaborative research network; PsA: psoriatic arthritis; PsC: cutaneous psoriasis.

\section{Operational Structure}

Dr. Chandran debated several designs that the CRN could use (Table 2) and how each of these designs would influence the CRN's operational structure. Based on the PsA BioDAM project, it is estimated that the cost per patient would be CAN\$4800 to collect clinical, blood, and imaging data [including magnetic resonance imaging (MRI)], with additional costs for bioinformatics support. Financial and academic compensation of sites for their contribution must therefore be finely mapped. The potential for reducing costs for some technologies must be balanced against inflation related to salaries, courier services, and some consumables. Converting the CRN's priority research areas into discrete work packages may help achieve this granularity of detail and aid logistical planning. Given that some other medical specialties are at more advanced stages of biomarker research and multinational collaboration (oncology, public health, cardiology, and nephrology), there may be value in liaising with such specialties.

A CRN committee structure was proposed and is detailed in Figure 1. The CRN structure could be centralized, with a few key sites directing work packages (as is done for multinational RCT); or federated, with semiautonomous decentralized sites that each send a proportion of their samples to the CRN (Figure 2).

\section{Funding Avenues and Requirements}

Professor Gladman, Professor Philip J. Mease, Jackie Anderson (representing Abbvie), and Lara Fallon (repre-

Table 2. Possible CRN designs.

\begin{tabular}{ll}
\hline Design & Observational vs interventional \\
& Registry vs RCT \\
& Cross-sectional vs longitudinal \\
& Inception vs cohort \\
& Personalized medicine \\
Participants & Eligibility criteria for cases \\
& Inclusion of control subjects, especially important for \\
laboratory and imaging biomarkers & Number \\
Gites & Geographical representation \\
& Targeting special populations who \\
& - have been underresearched to date \\
& - present with extreme phenotypes \\
& Closed vs open to new sites after commencement \\
& Recruitment cap for each site to improve external \\
& validity \\
& Biosamples contribution \\
& - mandatory for all sites vs some contributing \\
& clinical data only \\
Effective IT solutions that are robust over time & NGS-type storage of extremely large datasets \\
& Different systems for genomic vs imaging data \\
Data dictionary, to ensure consistency of terminology, \\
especially for clinical terms
\end{tabular}

CRN: collaborative research network; RCT: randomized controlled trials; IT: information technology; NGS: next-generation sequencing.

Personal non-commercial use only. The Journal of Rheumatology Copyright $\subset$ 2018. All rights reserved. 


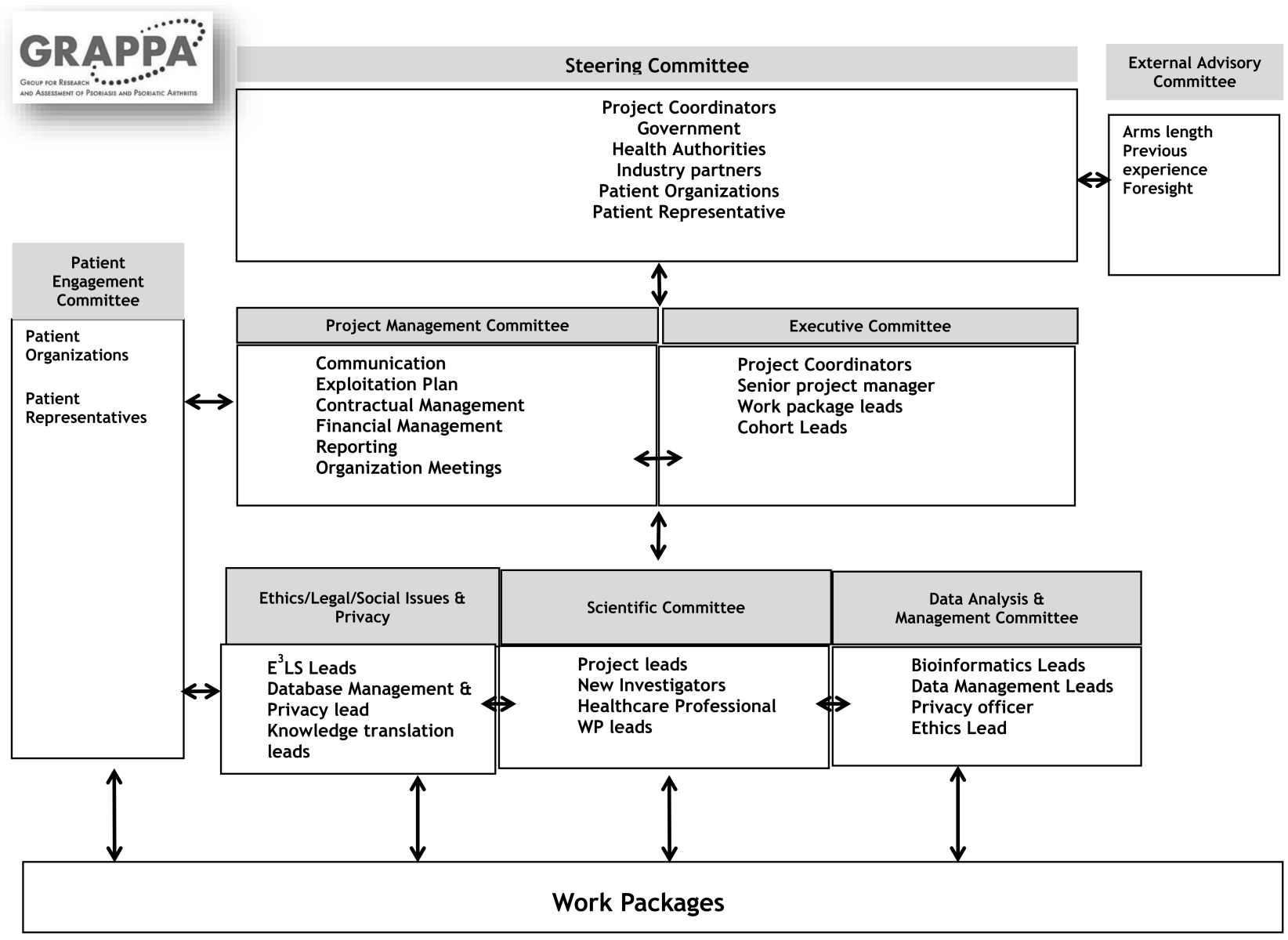

Figure 1. Possible committee structure for the Collaborative Research Network. E3 LS: Ethical, Environmental, Economic, Legal, Social Aspects; WP: work package.

senting Pfizer) participated in a panel discussion to identify potential funding avenues and related considerations. Several candidates to approach for funding were proposed, including the Foundation of NIH, which offers individual grants and requests for applications (RFA) and whose feedback on the PsA-BioDAM project was positive ("good structure, but would benefit from utilizing clinical data from existing RCTs and incorporating more imaging to help reduce the number of candidate blood biomarkers"); NIH through individual grants and RFA; European Union and UK agencies (IMI, NIHR, MRC, Wellcome); foundations, such as the Gates Foundation; local agencies, such as Arthritis Society and Arthritis Research UK; and private donors. The use of existing infrastructure should also be examined (e.g., Newfoundland genetics database supported by IBM).

Industry funding could take place through collaboration, investigator-initiated research grants, other grants, and requests for data and products. The former 2 options might best align with the current CRN remit. An example of requests for data and products is AbbVie's "Open Innovation Portal" ", which enables researchers to access AbbVie's pipeline products at various stages of development. Industry are optimally positioned and have the experience to contribute expertise in running multinational RCT, writing SOP for biosampling, and applying for and implementing ethical approvals and material transfer agreements (MTA) across international borders. Industry's criteria to fund and collaborate with the CRN would likely be determined by a project's scientific fit with a company's strategy and pipeline products. Additional factors may include applicant experience (centers and individuals), scientific rigor, project focus, robustness of project management, and the likelihood of success. A "private-public partnership" may be appealing to some companies, with active at-inception involvement in study design and database creation.

GRAPPA may prefer to have multiple, rather than single, industry partners. This might, however, affect IP agreements . Further, the funder's legal role and position will determine IP ownership. One solution might be for GRAPPA to own the IP, and if funded by multiple industry partners, existing partners would have the first option to commercialize the IP with GRAPPA. This approach would entail further consider- 


\section{CRN Architecture}

\section{Centralised}

- One or few key sites direct the program/work package

- Similar to a multi-centre study with $\mathrm{PI}$, coPIs and collaborators

- Interventional trials

- Centrally directed IRB process

- Data, IP etc. usually owned by PI

- Funding centrally raised and allocated

- MTAs between central site(s) and each contributing site

- Authorship to all contributors

- Timeline: defined

- Costs: Better defined
Federated

- Semi-autonomous de-centrally organized

- Each site owns data but stores/sends 'spare' samples to central site

- Observational studies

- Each site has its own IRB but consent allows sharing data

- Central repository only a custodian- no ownership

- MTA between all sites

- Each participating site applies to research committee to seek approval for access to samples, owns IP

- Ideal for discovery-validation studies

- Authorship depends on contribution; not for just providing data to repository

- Timeline: in perpetuity???

- Costs: May vary as new methods incorporated

Figure 2. Possible architecture of the CRN: centralized versus federated. CRN: Collaborative Research Network; PI: principal investigator; IP: intellectual property; MTA: material transfer agreements; IRB: institutional review board

ation regarding whether the CRN should be an "organization" or a "business," which is partly determined by the CRN's main remit.

\section{GRAPPA Member Survey}

Dr. Deepak R. Jadon and Dr. Carmel Stober reported the results of 2 surveys relating to the proposed CRN. The first survey disseminated to all 400 GRAPPA members in September 2016 was completed by 99 members, with 61 centers across 25 countries (and Hong Kong) wishing to join and contribute to the CRN (Table 3). In keeping with the proportional representation in GRAPPA, respondents included rheumatologists (81\%) and dermatologists (19\%).

Of centers wishing to participate, the following associated items were already being collected as part of existing research studies and could be contributed to the CRN: biosamples (53/99, 53\%; including serum 41, DNA 31, RNA 18, skin 14, PBMC 10, synovial fluid 10 , synovium 7 , urine 9 , and stool $2)$, clinical data $(80 / 99,81 \%$; including clinical phenotype 67 , demographics 67 , patient-reported outcome measures 36 , clinical examination indices 23 , comorbidity 50), imaging (55/99, 56\%; including plain radiographs 46 , MRI 26, ultrasound 17, and high-resolution peripheral quantitative computed tomography 1); and biosample processing SOP for sharing (36\%). The majority of respondents wanted to be both contributors and requestors of the CRN (70\%), while $23 \%$ wished to be contributors only.
A second survey was performed in June 2017 of the 61 respondents wishing to join the CRN to establish what resources centers already have in place, what biosamples and analyses are already being performed, to gather information on financing, scope project proposals, and identify already established SOP. There were 31 respondents, with a similar geographical distribution to the first survey. Ninety-seven percent of centers were willing to adopt harmonized SOP for biosample and data collection, and $94 \%$ of centers have personnel in place to facilitate collection. A total of 55\% of centers currently get consent from all patients attending clinic for future research studies, and $42 \%$ currently get consent from some but not all patients. A majority $(81 \%)$ of centers preferred ethical approval to be sought centrally by the CRN; although $61 \%$ already had ethical approval for local collection and $77 \%$ had ethical approval to send anonymized biosamples to other centers. Biosamples from other countries were already transferred or received by $45 \%$ of centers, with $23 \%$ of centers stating some restrictions in MTA across international borders and an average time of 3 months to set up an MTA. There was a majority preference for data to be entered into a shared database $(67 \%)$, with $42 \%$ of centers preferring paper and $45 \%$ eCRF. A requirement for external funding for clinical and biosample collection was stated by $55 \%$ of centers, as per the routes described earlier in this paper. The preference for biosample processing and storage was as follows: process biosamples locally and store locally

Personal non-commercial use only. The Journal of Rheumatology Copyright (c) 2018. All rights reserved. 
Table 3. Countries (including Hong Kong) with centers wishing to participate and contribute to the Collaborative Research Network.

\begin{tabular}{|c|c|}
\hline Country & No. Centers \\
\hline \multicolumn{2}{|l|}{ North America } \\
\hline USA & 19 \\
\hline Canada & 4 \\
\hline \multicolumn{2}{|l|}{ Europe } \\
\hline UK & 9 \\
\hline Germany & 3 \\
\hline Ireland & 2 \\
\hline Spain & 2 \\
\hline Turkey & 2 \\
\hline the Netherlands & 1 \\
\hline Belgium & 1 \\
\hline Croatia & 1 \\
\hline Iceland & 1 \\
\hline Italy & 1 \\
\hline \multicolumn{2}{|l|}{ South America } \\
\hline Brazil & 5 \\
\hline Argentina & 4 \\
\hline Colombia & 2 \\
\hline Peru & 1 \\
\hline Venezuela & 1 \\
\hline \multicolumn{2}{|l|}{ Middle East } \\
\hline Israel & 3 \\
\hline Bahrain & 1 \\
\hline \multicolumn{2}{|l|}{ Asia } \\
\hline Japan & 1 \\
\hline Hong Kong & 1 \\
\hline Singapore & 1 \\
\hline Taiwan & 1 \\
\hline China & 1 \\
\hline \multicolumn{2}{|l|}{ Africa } \\
\hline South Africa & 2 \\
\hline Australia & 2 \\
\hline
\end{tabular}

$(15 / 32 ; 47 \%)$; process centrally and store centrally $(12 / 32$; $38 \%)$; and process locally and store centrally $(5 / 32 ; 16 \%)$.

GRAPPA members had immense enthusiasm for the CRN . The community would prefer centralized ethics, samples to be locally processed and locally stored as part of a "virtual biorepository," and a centralized clinical database. The majority of centers are currently collecting serum and DNA. The collection of RNA could be promoted through the use of a simple system such as the PAXgene Blood RNA tube.

\section{Electronic Clinical Data Collection}

Dr. Alexis Ogdie led a discussion on electronic data collection options for the CRN. A balance must be struck between comprehensive data that would address research requiring well-characterized cohorts versus feasibility, so that data collection and entry are not too onerous for centers. The research question being addressed is an important determinant of the complexity of data required and may therefore be best stratified into "core" and "extended" datasets. It would be sensible to incorporate some extra margin to permit unplanned analyses. Data should ideally be organized into tables: patient demographics, phenotype, visit information, samples collected, examination findings, diagnoses, comorbidities, social history, medications, etc., and with patient-reported outcomes for each domain. It will be important to standardize terminology for medications, dosing, and durations. It should be emphasized that "standardized data elements" does not mean standardized data collection or definitions. There can be much disparity between electronic medical records systems. Attention must be given to license fees and copyright issues relating to some clinical indices and patient-reported outcome measures. The storage of data both centrally and locally will improve clinical governance by ensuring integrity. Based upon all of these factors, it was recommended that the $\mathrm{CRN}$ collect data into a centralized database.

\section{Authorship}

Given the number of potential contributors to the CRN, Dr. Philip S. Helliwell led discussions on how authorship of publications relating to the CRN could be approached. There is certainly a need for an a priori publication policy, including a process for resolving disputes and appeals. The International Committee of Medical Journal Editors guidelines for publishing manuscripts ${ }^{6}$ or the AMP Group Authorship Guidelines are models that could be implemented. The steering committee of the $\mathrm{CRN}$ or of a particular work package could agree on first and senior author roles. Other authors not to be overlooked include PRP, statisticians, sponsors, and clinical, laboratory, and imaging data collectors. Thresholds for clinical case contribution to the CRN may need to be set to attain authorship roles. A data sharing agreement would add value to the CRN's operation.

\section{Discussion}

The Classification of Psoriatic Arthritis ${ }^{7}$ initiative is an exemplar of how 32 centers worldwide can collaborate to achieve great results through GRAPPA. Four candidate flagship research areas for the CRN include (1) predictors of treatment response in PsA and PsC, thereby permitting personalized and stratified medicine approaches; (2) predictors of structural damage and disease severity, linking with the existing PsA BioDAM project; (3) predictors of PsC progressing to PsA, thereby enabling earlier intervention and possibly even halting progression to PsA; and (4) comorbidity prevalence and effect on clinical outcomes in psoriatic disease.

A hybrid model with a centralized eCRF and harmonized SOP across sites based on rigorous validation appears to be the best approach going forward. Investigators must be

Personal non-commercial use only. The Journal of Rheumatology Copyright @ 2018 . All rights reserved. 
engaged through the collection of high-quality data that increases the likelihood of securing funding, opportunities to fund projects using samples from their sites, and nurturing other collaboration opportunities. PRP should be involved at every stage of the CRN's development. It may be sensible to further develop and refine existing projects such as the PsA BioDAM, into which much expertise and time has already been invested. The IPART and RA BioDAM projects will inform the CRN's centralized eCRF, biosample collection, and operational logistics. Validated SOP must be harmonized across centers, with training programs developed for both faculty and trainees to aid their correct implementation, scientific rigor, and reproducibility. A team of methodologists, biostatisticians, and "big data" scientists must be assembled to develop and implement novel analytic approaches for the CRN. While partnership with industry is fundamental, more stable longterm funding through national and government-related agencies should be sought as a priority.

The collaboration and momentum provided by a GRAPPA-CRN will offer more than the sum of its individual contributing centers. A CRN will permit high-quality research that can more effectively address questions pertinent to patients, clinicians, scientists, industry, and governments.

\section{REFERENCES}

1. National Institutes of Health. Accelerating Medicines Partnership (AMP). Overview. [Internet. Accessed February 14, 2018.] Available from: www.nih.gov/research-training/ accelerating-medicines-partnership-amp

2. National Institute of Arthritis and Musculoskeletal and Skin Diseases (NIAMS). Roundtable on gaps and emerging opportunities in psoriatic arthritis. [Internet. Accessed February 26, 2018.] Available from: www.niams.nih.gov/about/meetings-events/ roundtables/forum-gaps-psoriatic

3. National Institutes of Health. Accelerating Medicines Partnership (AMP). Autoimmune diseases of rheumatoid arthritis and lupus. [Internet. Accessed February 14, 2018.] Available from: www.nih.gov/research-training/accelerating-medicines-partnershipamp/autoimmune-diseases-rheumatoid-arthritis-lupus

4. Segurado OG, Sasso EH. Vectra DA for the objective measurement of disease activity in patients with rheumatoid arthritis. Clin Exp Rheumatol 2014;32 Suppl 85:S-29-34.

5. Abbvie Open Innovation Portal 2017 [Internet. Accessed February 14, 2018.] Available from: www.openinnovation.abbvie.com

6. ICMJE. International Committee of Medical Journal Editors Guidelines for Publishing Manuscripts. [Internet. Accessed February 14, 2018.] Available from: www.icmje.org

7. Taylor W, Gladman D, Helliwell P, Marchesoni A, Mease P, Mielants $\mathrm{H}$, et al. Classification criteria for psoriatic arthritis: development of new criteria from a large international study. Arthritis Rheum 2006;54:2665-73. 